\title{
THE LIPIDS OF MICROALGAE
}

\section{LIPIDS OF THE DRY BIOMASS OF Monochrysis lutheri}

\author{
T. V. Khomova, S. D. Gusakova, and A. I. Glushenkova
}

UDC 547.915:665.3

Continuing the investigation of microalgae [1], we have determined some indices of the lipid composition of the freeze-dried biomass of Monochrysis lutheri. The biomass was grown in a laboratory glass-tube cultivator in a nutrient medium containing all the biogenic elements with all-day illumination, temperature $26^{\circ} \mathrm{C}, \mathrm{pH} 7.0{ }^{*}$

The yield of lipids on extraction of the biomass with chloroform-methanol $(2: 1)$ was $38.6 \%(386 \mathrm{mg} / \mathrm{g}$ of biomass).

Neutral and polar classes were identified among the lipids. The bulk of the neutral lipids (TLC on silica gel L 5/40 and Silufol in the hexane-diethyl ether (8:2,6:4, and 8:7) systems with revelation by $\mathrm{H}_{2} \mathrm{SO}_{4}$ and $\mathrm{I}_{2}$ ) consisted of triacylglycerols, which were accompanied by hydrocarbons, carotenoids (UV spectrum, $\lambda_{\max }$ hexane, $n m: 406,424,445,473$ ), esters of sterols and triterpenoids with fatty acids, fatty acid methyl esters, free fatty acids, chlorophylls and their derivatives (UV spectrum, $\lambda_{\max }$ acetone, $\mathrm{nm:}$ : 406, 423, 470, 502, 536, 564, 602, 653, 660), sterols, and triterpenols. Among glycosides (TLC on silica gel in the chloroform-methanol-water (65:25:4) system with $\alpha$-naphthol as the revealing agent) there were steryl glycosides and their esters, sulfoquinovosyldiacylglycerols, and monogalactosyl- and digalactosyldiacylglycerols, with a predominance of the latter. Among phospholipids (TLC under the same conditions as for the glycosides, with revelation by the Dragendorff and Vas'kovskii reagents and ninhydrin) - phosphatidylglycerol, phosphatidylethanolamine, phosphatidylcholine, phosphatidylserine, phosphatidylinositol, lysophosphatidylcholine, and phosphatidic acid - lecithin predominated.

The amount of unsaponifiables among the lipids was (mg/g of lipids) 88.6 , of carotenoids 25.04 , of chlorophylls 27.59 , and of fatty acids 250.7 .

According to TLC, the composition of the fatty acids was (\% on the weight of the acids): 8:0-w, 10:0-w, 12:0$0.1,14: 0-3.6,14: 1-3.6,14: 1-0.1,14: 2-0.2,16: 0-41.6,16: 1-29.9,16: 2-0.7,16: 3-0.2,18: 0-0.6,18: 1-0.6,18: 1-2.0,18: 2-2.5$, $18: 3-0.1,20: 0-1.2,20: 4-4.0,20: 5-13.2$. The presence of 17 fatty acids was confirmed by the mass spectrometry of their methyl esters, with the additional identification of the following fatty acids 15:3, 16:4, 17:2, 17:3 and 19:2 $\left(\mathrm{M}^{+} 250,262,280,278\right.$, 308; $[\mathrm{M}-31]+219,231,249,247,277 ;[\mathrm{M}-74]+176,188,206,204,234$, respectively).

On comparing the indices of the freeze-dried Monochrysis biomass grown in a glass-tube cultivator in sea water with natural illumination (in Anapa) that we studied previously and the biomass of the present Monochrysis sample, it can be seen that approximately equal amounts of lipids were synthesized in them. The compositions of the lipids of the two samples were close but they differed in the amounts of polyunsaturated acids, which possess a high biological activity [2] (10.8\% less in the present sample).

\section{REFERENCES}

1. T. V. Khomova, S. D. Gusakova, L. G. Mezhlum'yan, and A. I. Glushenkova, Khim. Prir. Soedin. No. 4, 507 (1993).

2. D. J. Kyle. J. Am. Oil Chem. Soc., 64, 1251 (1987).

*The biomass was supplied for study by G. S. Skotnikova of the All-Russian Scientific Research Institute for Biotechnology (Moscow).

Institute of the Chemistry of Plant Substances, Uzbekistan Academy of Sciences, Tashkent. Translated from Khimiya Prirodnykh Soedinenii, No. 6, pp. 899-900, November-December, 1993. Original article submitted July 6, 1993. 